\title{
Firenze e Pistoia: Governo del territorio e fazioni cittadine*
}

\section{Luca Vannini}

Università di Firenze

SOMMARIO: Durante il processo di edificazione del proprio dominio territoriale, Firenze affrontò le peculiarità di ciascuna città soggetta attuando scelte strategiche differenti. Nel caso della città di Pistoia le autorità fiorentine seppero modulare nel tempo interventi specifici al fine di gestire e governare il territorio. Ad un primo momento (1329-1376) in cui alla comunità pistoiese fu offerta una sorta di 'protezione' politico-militare, tradottasi ben presto in una progressiva erosione della sovranità politica, seguì, dopo il 1376, la formale istituzionalizzazione del bipartitismo cittadino, che contribui a congelare il conflitto tra le fazioni e consentì a Firenze un più agevole controllo della vita politica cittadina. Dopo l'avvento del regime mediceo le dinamiche politiche delle parti pistoiesi furono gestite dalla potente famiglia fiorentina in modo quasi esclusivo, tanto che dopo il 1458 il regime bipartitico poté di fatto essere abolito, giungendo infine, in epoca laurenziana, ad un vero e proprio 'monopolio' del patronato. Questa terza fase delle relazioni tra Firenze e Pistoia, conclusasi all'alba del crollo del regime mediceo nel 1494, lasciò in Pistoia un tale vuoto di potere che alla fine del secolo la lotta di fazione non poté che riesplodere ancora più cruenta.

Parole chiave: Firenze; Pistoia; dominio territoriale; fazioni; bipartitismo; patronato.

* Archivio di Stato di Firenze, ASF: Acquisti e doni; Capitoli; Consulte e pratiche; Mediceo avanti il Principato; Provvisioni; Statuti delle comunità autonome e soggette. Archivio di Stato di Pistoia, ASPt: Comune, Provvisioni; Consigli, provvisioni e riforme. Biblioteca Forteguerriana di Pistoia, BFP: Fondo Forteguerriano, C 225 (Buoni, Bastiano, Istoria di Pistoia dal 1499 al 1504). Biblioteca Marciana di Venezia, BMV: Manoscritti, CL, It., VI 197 (5803) (Melocchi, Jacopo, De’ casi di Pistoia). 


\section{Florencia y Pistoia: gobierno territorial y facciones citadinas}

RESUMEN: Durante el proceso de expansión territorial y de construcción de un dominio a escala subregional, la ciudad de Florencia fue consciente de las especificidades locales de cada una de las comunidades sometidas y por ello llevó a cabo estrategias diferentes y opciones políticas diferentes. En el caso de la ciudad de Pistoia —no sometida formalmente hasta después de 1401, a decir verdad-, y de sus «malditas facciones», las autoridades florentinas fueron capaces de aplicar en el momento preciso diferentes politicas e instrumentos para la gestión y el gobierno del territorio. A un primer momento (1329-1376), cuando a la comunidad de Pistoia se le ofreció una especie de «protección» político-militar, que se tradujo de todas formas y enseguida en una progresiva erosión de la soberanía política (al menos desde la segunda mitad del siglo XIV), le siguió, después de 1376, la institucionalización formal del bipartidismo ciudadano, que contribuyó a enfriar el conflicto que existía entre las facciones de los Panciatichi y de los Cancellieri e hizo posible que Florencia ejerciera un mayor y ágil control de la vida política de Pistoia. Una vez instaurado el régimen mediceo y, de modo especial, a partir de la segunda mitad del siglo XV, los Medicis comenzaron, mediante sus bien conocidas redes clientelares y de patronato, a controlar casi totalmente las dinámicas de las facciones de Pistoia, hasta tal punto que después del año 1458 , el régimen bipartito pudo ser abolido de facto, alcanzándose, finalmente, en época de Lorenzo, un verdadero y auténtico 'monopolio' del patronato sobre las partes de Pistoia. Esta tercera fase de las relaciones entre Florencia y Pistoia, finalizada cuando se desmoronó el régimen mediceo en 1494, dejó en Pistoia un enorme vacio de poder que dio lugar a que la lucha de facción fuese cada vez más cruenta, volviéndose a producir los trágicos acontecimientos que ya a principios del siglo XV habían conducido a la decidida intervención de Florencia, ratificada por la sumisión de la ciudad de Pistoia, en el mismo momento en el que se concedió a Florencia el vicariato imperial sobre todas las comunidades sometidas de su territorio.

\section{PAlabras ClaVE: Florencia; Pistoia; dominio territorial; facción; bi- partidismo; patronato.}

\section{Florence and Pistoia: territorial government and city factions}

ABSTRACT: During the process of its territorial dominion's building, Florence faced the peculiarities of each subject town by implementing different strategic choices. In the case of the city of Pistoia the Florentine authorities were able to modulate specific interventions over time in order to manage and govern the territory. After a first period (1329-1376) in which a sort of political and military 'protection' was offered to the community of Pistoia - soon turned into a gradual erosion of political sovereignty- the formal institutionalization of city bipartisanship followed and helped to freeze the conflict between the factions, giving to Florence an easier control over city's political life. After the advent of the Medici regime the political dynamics of the parts of Pistoia were managed by the powerful Florentine family almost exclusively, so that after 1458 the bipartisan re- 
gime could in fact be abolished, coming finally in the Laurentian age to a real 'monopoly' of patronage. This third phase of the relationships between Florence and Pistoia, which ended at the dawn of the collapse of the Medici regime in 1494, left in Pistoia such a power void that the factional struggle broke out again even more bloody at the end of the century.

KEY WORDS: Florence; Pistoia; territorial dominion; factions; bipartisanship; patronage.

\section{INTRODUZIONE}

Solevano li antichi nostri, e quelli che erano stimati savi, dire come era necessario tenere Pistoia con le parte e Pisa con le fortezze; e per questo nutrivano in qualche terra loro suddita le differenzie, per possederle più facilmente. Questo, in quelli tempi che Italia era in uno certo modo bilanciata, doveva essere ben fatto: ma non credo già che si possa dare oggi per precetto; perché io non credo che le divisioni facessino mai bene alcuno: anzi è necessario, quando el nimico si accosta, che le città divise si perdino subito, perché sempre la parte più debole si aderirà alle forze esterne e l'altra non potrà reggere ${ }^{1}$.

Come suggerito dal celebre passo machiavelliano, nell'orizzonte della costruzione di un dominio territoriale di ambito subregionale, Firenze si trovò costretta a fronteggiare specificità locali che la obbligarono a mettere in campo scelte strategiche differenti. Nel caso della città di Pistoia le «sectas et sedictiones bestiales», così come le definisce l'umanista Sozomeno ${ }^{2}$, sacerdote e canonico della cattedrale di Pistoia che visse tra il 1387 e il 1458, quegli elementi cioè che più di ogni altro contribuirono a caratterizzare l'immagine della città tra Tre e Quattrocento, costituirono per Firenze sia un'opportunità per imporre più facilmente la propria dominazione, che un problema di ordine e sicurezza interna con cui misurarsi costantemente nel corso del XV secolo. Questa duplice valenza della dimensione bipartitica e fazionaria della vita politica pistoiese servì spesso quale ragione determinante e strumento di giustificazione dell'intervento fiorentino a partire almeno dall'ultimo decennio del XIII secolo, quando, proprio a causa della manifesta incapacità nel risolvere le divisioni cittadine e le lotte di fazione tra guelfi bianchi e guelfi neri, fu concessa a Firenze una balìa per governare la città di Pistoia dal 1296 al $1301^{3}$. Anche in precedenza vi erano stati comunque altri interventi fiorentini

\footnotetext{
1 MACHIAVELLI, 1995, cap. XX: 140.

2 SOZOMENO, 1908: 53.

3 CHERUBINI, 1998: 41-88.
} 
nel 1239, 1254 e 1258, per evitare che la città di Pistoia aderisse a schieramenti e fronti contrari alla città del giglio ${ }^{4}$.

\section{DAI PRIMI CAPITOLI DEL 1329 ALL'ISTITUZIONALIZZAZIONE DEL BIPARTI- TISMO}

Nel primo quarto del Trecento vi furono conflitti ricorrenti tra la Firenze dei guelfi neri e la Pistoia dei guelfi bianchi; nel 1325 Castruccio Castracani ottenne la signoria della città, e la tenne fino al 1328, anno della sua morte. I primi Capitoli tra Firenze e Pistoia furono così siglati l'anno successivo (24 maggio 1329): in occasione della stipulazione della pace la città di Pistoia si obbligava ad obbedire al papa e alla Chiesa, a riammettere tutti i guelfi che erano stati esiliati e colpiti da bando, a cancellare tutti i procedimenti pendenti nei confronti di cittadini fiorentini, a concedere a Firenze il possesso dei castelli di Montemurlo e Tizzana, e ad impegnarsi a non accogliere più nel suo territorio ribelli o nemici della Chiesa o del comune di Firenze 5 .

Nel 1331, con una balìa concessa per un anno e successivamente rinnovata fino al 1340, gli organi del comune di Pistoia assegnarono a Firenze la «libera custodia della città, contado e distretto», in deroga ai vigenti statuti ed ordinamenti, affinché fosse provveduto "circa securitatem et pacificum statum» della città 6 . Firenze stabilì in Pistoia una guarnigione armata e un capitano di custodia, il quale coesistette con il capitano del popolo (conservator pacis) fino al 1367, e in seguito ne integrò le funzioni e lo sostituì completamente ${ }^{7}$. I pistoiesi continuarono invece ad eleggere i propri podestà, anche se in momenti di particolare tensione furono scelti per ricoprire tale carica dei fiorentini. Le riforme e gli interventi politici di questi anni rappresentano perciò la prima importante cesura nella vita politica della città di Pistoia: Firenze comincia a ridisegnarne le strutture istituzionali secondo i propri principali interessi di città dominante. Nel 1331, infatti, vengono soppresse le corporazioni delle arti e nel 1332 le compagnie rionali ${ }^{8}$.

Pistoia riuscì a riguadagnare una sostanziale autonomia politica soltanto in seguito alla breve parentesi costituita in Firenze dalla signoria del Duca d'Atene (1342-1343), giungendo persino ad allontanare i fiorentini dai

4 CHERUBINI, 1998: 54-60, 70. HERLIHY, 1972: 250-252.

5 Capitoli del Comune di Firenze, vol. 1, registro I, doc. n. 4 del 24 maggio 1329 (cc. 4v-9r): 5-7. $11 \mathrm{v}): 8$.

6 Capitoli del Comune di Firenze, vol. 1, registro I, doc. n. 6 del 26 luglio 1331 (cc. 10v-

7 ASPt, Comune, Provvisioni, 14, c. 6r.

8 CHERUBINI, 1998: 71. 
castelli di Santa Barbara e di Serravalle. Furono approvati nuovi statuti e fu richiesto un nuovo giuramento di fedeltà alle comunità territoriali del contado e del distretto pistoiese. A partire dal 1351, però, in seguito al maturare del conflitto antivisconteo che vide protagonista Firenze contro l'Arcivescovo Giovanni, signore di Milano dal 1329 al 1354, Pistoia rientrò nuovamente sotto l'influenza fiorentina. Il Visconti aveva acquistato per la somma di duecentomila fiorini la città di Bologna dalla famiglia Pepoli (ottobre 1350), e si preparava a minacciare da vicino la stessa Firenze. Le contromosse fiorentine si concretizzarono nell'acquisto e nella successiva occupazione di Prato, e nel tentativo di procedere allo stesso modo con Pistoia. È in questa occasione che Firenze dovette tornare a confrontarsi con le «maledette fazioni» pistoiesi. Negli ultimi anni, infatti, le famiglie dei Panciatichi e dei Cancellieri si erano apertamente contrastate per il governo della città, spartendosi la gestione degli uffici pubblici grazie ad una rete di clientele che coinvolgeva una trentina di altre famiglie pistoiesi. Sia i Panciatichi che i Cancellieri potevano inoltre vantare importanti alleanze con eminenti famiglie fiorentine 9 .

Dal 1329 le famiglie magnatizie di Pistoia erano state escluse dagli uffici pubblici, ma, in virtù dell'aiuto prestato ai fiorentini contro Castruccio, alcune di esse (Panciatichi, Gualfreducci, Muli) godevano ancora della partecipazione alla vita politica pistoiese. I Panciatichi avevano cominciato così a prevalere in città, nonostante lo statuto del 1344, successivo alla cacciata del Duca d'Atene, li avesse nuovamente esclusi dalle cariche pubbliche ${ }^{10}$. Riccardo Cancellieri, cercando di impossessarsi di Pistoia intorno al 1350, mosse una decisa offensiva contro i Panciatichi, i quali però furono in grado di guidare la reazione dell'intera città e lo costrinsero a rifugiarsi nel castello di Marliana ${ }^{11}$. I fiorentini, temendo che i Panciatichi (di tradizione ghibellina) potessero appoggiare il Visconti, convinsero la città ad accettare la difesa e il sostegno di truppe armate fiorentine, per potersi meglio difendere dall'offensiva del Cancellieri. In questo senso i Capitoli sottoscritti il 6 maggio $1351^{12}$ costituirono un ulteriore passo sulla via del controllo politico di Pistoia da parte di Firenze. La città non fu direttamente annessa, come era successo con Prato, ma si ricorse nuovamente all'istituto della balìa, cercando di ottenere la pacificazione delle fazioni e il loro sostanziale equilibrio, in modo che nessuna di esse potesse più prevalere sull'altra. Nei Capitoli i sindaci del comune di Firenze promettono comunque espressamente che: «non occupabitur libertas nec iurisditio dicti Communis Pistorii», così come il sindaco del comune di

9 NERI, 1999: 5-6.

10 CHERUBINI, 1998: 71, nota n. 164.

11 FIORAVANTI, 1968: 316.

12 Capitoli del Comune di Firenze, vol. 1, registro I, doc. n. 14 del 6 maggio 1351 (cc. 19r-23r): 11-12. 
Pistoia promette: «quod dicta civitas Pistorii reformabitur in vero statu libero populari et guelfo et in vera Parte Guelfa» ${ }^{13}$. Secondo questa nuova balìa, che avrà durata quindicennale e sarà rinnovata per ulteriori quindici anni nel $1365^{14}$, Firenze può imporre in Pistoia anche la presenza di un podestà fiorentino (capitaneus populi et communis), nella persona di Filippo di Duccio Magalotti, a partire dal 21 dicembre 1351.

Osserva a questo proposito Francesco Neri:

A partire dal 1351 iniziò un lento processo di intervento sempre più diretto da parte delle autorità fiorentine nei confronti di Pistoia, che si attuò innanzitutto svuotando di significato le strutture amministrative e giuridiche del Comune, in alcuni casi lasciate sopravvivere ma ridotte nella capacità di autonoma scelta ${ }^{15}$.

Le stesse autorità fiorentine continuavano del resto a ribadire la volontà di non usurpare la libertà pistoiese, come testimoniato dal giuramento prestato da sessanta cittadini fiorentini in occasione della proroga della balìa e dei Capitoli del 1351: «Sessanta Cittadini fiorentini giurarono 'ad sancta Dey evangelia, non occupare libertatem vel iurisditionem dicti Communis Pistorii', salvi sempre i capitoli e patti suddetti» ${ }^{16}$. Ma la realtà delle cose evolveva in maniera alquanto differente.

Dal 1367, infatti, le funzioni del capitano del popolo passarono al capitano di custodia (ovviamente sempre un cittadino fiorentino), e il podestà venne escluso dal Consiglio generale del popolo e del comune, che di fatto passò sotto il diretto controllo del medesimo capitano di custodia. Tali provvedimenti, contenuti poi nella riforma generale degli uffici fatta dagli ambasciatori fiorentini in data 23 novembre $1373^{17}$, riguardarono anche la riduzione dei membri del principale organo deliberativo, il Consiglio generale sopra menzionato, che furono portati a 128 (32 membri per porta) ed estratti da borse predisposte sotto la tutela e la supervisione dei fiorentini. Fu nuovamente consentito ai magnati l'accesso alle cariche pubbliche, in modo da fare emergere una nuova oligarchia direttamente legata alle principali famiglie fiorentine; fu istituita una nuova magistratura, gli Octo boni homines (portati a dodici nel 1376), con il compito di assistere gli Anziani nel proporre questioni e deliberazioni al Consiglio. Anche nelle rubriche di questi Capitoli del 1373 i

13 Capitoli del Comune di Firenze, vol. 1, registro I, doc. n. 14 del 6 maggio 1351 (cc. 19r-23r): 12.

14 Capitoli del Comune di Firenze, vol. 1, registro I, doc. n. 16 del 31 ottobre 1365 (cc. 23r-24v): 13.

15 NERI, 1999: 7.

16 Capitoli del Comune di Firenze, vol. 1, registro I, doc. n. 17 del 31 ottobre 1365 (cc. $24 \mathrm{v}-25 \mathrm{v}): 13$.

17 Capitoli del Comune di Firenze, vol. 1, registro I, doc. n. 18 del 23 novembre 1373 (cc. 27r-37r): 13-18. 
fiorentini non mancarono di fornire ulteriori attestazioni della loro buona volontà nei confronti delle libertà pistoiesi: «Tutti i capitani della custodia siano tenuti, a pena di lire 500 da applicarsi al Comune di Firenze, a giurare, prima che vadano in ufficio, nelle mani dei Priori delle Arti e Gonfaloniere di giustizia, di conservare la città di Pistoia, contado e distretto in quella libertà e stato che gode al presente, e nella devozione e 'filiatione' del Comune di Firenze» ${ }^{18}$. Il controllo politico di Firenze assumeva nella retorica della formulazione cancelleresca la forma di una 'tutela' paterna, volta unicamente al rispetto della devozione filiale di Pistoia ed interessata al mantenimento della salute politica della città, ottenuta attraverso la promozione della giustizia, della libertà e dell'equalitas ${ }^{19}$.

$\mathrm{Fu}$ forse proprio in nome di quest'ultima, intesa però dai fiorentini come uguaglianza ed equilibrio dell'autorità e del peso politico esercitato in Pistoia dalle due fazioni di Panciatichi e Cancellieri, che nel 1376 quattro ambasciatori fiorentini, insieme al capitano di custodia, annullarono la riforma degli uffici proposta da un comitato di ventiquattro cittadini pistoiesi e ne approvarono un'altra che, di fatto, portava esplicitamente all'istituzionalizzazione delle parti ${ }^{20}$. Furono, infatti, raddoppiate le borse per gli eleggibili agli uffici, in modo che ogni porta della città di Pistoia potesse averne due, una che contenesse i nominativi degli aderenti alla parte panciatica (compagnia di San Paolo) e l'altra quelli degli uomini fedeli alla parte cancelliera (compagnia di S. Giovanni), per un totale di otto borse per le quattro porte cittadine.

Il disegno politico della dominante non potrebbe apparire a questo punto più chiaro: attraverso l'istituzionalizzazione delle fazioni Firenze mira alla creazione di un equilibrio politico che si traduca nei fatti in una situazione di reciproca deterrenza tra le parti pistoiesi, e che consenta perciò un maggior controllo e una più forte influenza dell'autorità fiorentina. Una volta istituzionalizzate e rese legittime, le 'sette' in quanto tali possono anche venire denunciate e vietate, come si legge nell'ultima rubrica dei Capitoli del $1376^{21}$.

E' importante notare che l'istituzionalizzazione del bipartitismo cittadino, strumento di cui Firenze si servirà per governare Pistoia fino al 1457, veniva

18 Capitoli del Comune di Firenze, vol. 1, registro I, doc. n. 18 del 23 novembre 1373 (cc. 27r-37r): 17.

19 «Quo circa Commune Florentie, tamquam pius pater de salute Pistoriensium ac eorumdem libertate sollicitum, ad hoc ut equalitas in ipsius civitatis regimine observetur, vigeatque iustitia, sine quibus civitates et regna regi et conservari nequeunt»: Capitoli del Comune di Firenze, vol. 1, registro I, doc. n. 18 del 23 novembre 1373 (cc. 27r-37r): 14.

20 Capitoli del Comune di Firenze, vol. 1, registro I, doc. n. 21 del 24 aprile 1376 (cc. $39 \mathrm{v}-45 \mathrm{v}): 19-21$.

21 «25. Qualunque setta, e il titolo e nome di setta, sia riprovata e vietata in Pistoia; né alcuno osi chiamarsi d'alcuna setta»: Capitoli del Comune di Firenze, vol. 1, registro I, doc. n. 21 del 24 aprile 1376 (cc. 39v-45v): 21. 
portata a compimento «in un periodo in cui le principali famiglie pistoiesi si stavano collegando sempre più strettamente alle famiglie del ceto dirigente fiorentino» ${ }^{22}$. Emblematico a questo proposito il caso di Giovanni Novello Panciatichi, che nel 1375 sposò Piera di Pepo degli Albizzi e nel 1388 riuscì a farsi eleggere cavaliere della repubblica fiorentina ${ }^{23}$. Nello stesso tempo, mentre venivano rafforzati legami clientelari e il patronato fiorentino assumeva un ruolo egemone sul controllo delle famiglie e fazioni pistoiesi, Firenze, che dal 1373 aveva stabilito che anche il capitano della montagna pistoiese dovesse essere un cittadino fiorentino e dovesse risiedere a Cutigliano ${ }^{24}$, estendeva la propria autorità anche sulla gestione e sul governo del contado e della montagna di Pistoia, luoghi strategici per il controllo dei valichi appenninici e delle vie di comunicazione con la Lombardia.

Crebbero altresì in questo periodo le ingenti somme di denaro che Firenze si vide costretta a richiedere alle comunità soggette, compresa Pistoia, per far fronte alle continue guerre contro la Milano viscontea, specie alla fine del XIV secolo quando lo scontro con Gian Galeazzo minacciava seriamente la sopravvivenza stessa della repubblica fiorentina. Nei momenti più difficili di questo conflitto anche la carica di podestà di Pistoia dovette essere affidata unicamente a cittadini fiorentini. Con l'ulteriore riforma generale degli uffici del $1383^{25}$ le famiglie dei Panciatichi e dei Ricciardi furono nuovamente escluse dalla partecipazione agli uffici pubblici, e furono confermati e ampliati i poteri del capitano di custodia e con essi, ovviamente, l'autorità e la giurisdizione di Firenze sopra la vita politica pistoiese.

\section{GLI SCONTRI DI FAZIONE DEL 1400-01. L'INTERVENTO FIORENTINO E LA FORMALE SOTTOMISSIONE DELLA CITTÀ}

L'equilibrio tra le parti di Pistoia, artatamente imposto dalla dominazione fiorentina, subì un primo grande colpo nel biennio 1400-1401. Le tensioni tra Panciatichi e Cancellieri riesplosero all'alba del nuovo secolo ed ebbero origine da controversie matrimoniali riguardanti importanti alleanze familiari e clientelari $^{26}$. Senza addentrarci troppo, per brevità d'esposizione, nello svolgimento dei fatti (per i quali si rimanda alle opere citate in nota), possiamo

22 NERI, 1999: 8.

23 NERI, 1999: 9. CONNELL, 53 (1991): 533, nota n. 35.

24 FIORAVANTI, 1968: 322-323.

25 Capitoli del Comune di Firenze, vol. 1, registro I, doc. n. 24 del 28 marzo 1383 (cc. 47r-56v): 22-24.

26 Cronache di ser Luca Dominici, 1933-1939, vol. 2: 28-29. FIORAVANTI, 1968: 343. HERLIHY, 1972: 230. DE ANGELIS, 1995, vol. 4: 1159. 
affermare che furono subito coinvolte anche le autorità fiorentine, prima, nella persona del capitano di custodia Alberto di Cipriano Mangioni, che giunse a condannare a morte Niccolò di Pandragone Cancellieri27, e successivamente anche i Priori di Firenze i quali, a seguito delle pressioni di Riccardo Cancellieri, fecero annullare la sentenza capitale ${ }^{28}$.

Fino Cantasanti, complice insieme al Pandragoni dell'accusa di parzialità nei confronti dei Panciatichi rivolta al capitano di custodia, venne poi arrestato dalle autorità fiorentine, e, sotto tortura, rivelò che i Cancellieri avevano ordito una congiura che doveva portare all'eliminazione di Giovanni Panciatichi e all'assunzione del governo della città di Pistoia, costringendo Firenze alla legittimazione del suo potere sotto la minaccia di schierarsi apertamente con Gian Galeazzo Visconti. La notizia si diffuse in Pistoia la mattina del 4 agosto 1401, e i Cancellieri furono costretti ad abbandonare la città per sfuggire alla reazione dei Panciatichi e delle autorità fiorentine. I Panciatichi potevano contare sull'appoggio di eminenti famiglie fiorentine (Pitti, Castellani, Vettori, Guasconi e Albizzi), mentre i Cancellieri erano legati ad alcune famiglie magnatizie (Ricci, Acciaiuoli, Medici) e ad altre famiglie di recente stato popolare (Frescobaldi, Cavalcanti, Bardi, Tornabuoni), che si trovavano in quel momento in posizione di ostilità nei confronti del regime fiorentino, essendo alcuni esponenti dei Medici e dei Ricci addirittura in esilio ${ }^{29}$. I Cancellieri rimasti a Pistoia furono catturati mentre Riccardo ed altri fuggirono nella montagna pistoiese ed occuparono la rocca della Sambuca ${ }^{30}$.

Questa prima grande rivolta all'autorità e al dominio di Firenze, tale da far crollare in un sol colpo il complesso equilibrio politico costruito in Pistoia dalla dominante nei decenni precedenti, oltre agli attriti e agli odi delle due fazioni di Panciatichi e Cancellieri, avrà sicuramente tratto alimento anche dalla difficile situazione congiunturale che entrambe le città di Pistoia e Firenze stavano attraversando all'alba del XV secolo. Pistoia si era infatti da poco ripresa dalla grande pestilenza del 1400, mentre Firenze viveva la fase più pericolosa e drammatica del conflitto antivisconteo, tanto che poté scampare la sua rovina unicamente a causa della morte di Gian Galeazzo Visconti avvenuta nel 1402. E' altresì vero, comunque, che la rivolta pistoiese consentì a Firenze di portare avanti un intervento deciso e risolutore, e permise così la definitiva sottomissione della città di Pistoia al dominio territoriale fiorentino.

Il 4 luglio 1401 Firenze aveva ricevuto dall'imperatore Roberto di Baviera il vicariato imperale sopra tutte le comunità territoriali soggette della Tosca-

27 ASPt, Comune, Provvisioni, 27, c. 9v, delibera consiliare del 1 marzo 1401.

28 Cronache di ser Luca Dominici, 1933-1939, vol. 2: 30.

29 Cronache di ser Luca Dominici, 1933-1939, vol. 2: 10-12. MILNER, 2002: 415.

30 ASF, Consulte e pratiche, I (1401), passim. Le Consulte e Pratiche della Repubblica fiorentina nel Quattrocento (1401), 1981, vol. 1. 
na, inclusa ovviamente Pistoia ${ }^{31}$. La sanzione de iure del dominio fiorentino consentiva la più totale libertà d'azione nei fatti della rivolta di Pistoia e nello scontro di fazione allora in atto. Leggiamo nei Capitoli della formale sottomissione che i Signori e i Collegi

Decreverunt quod civitas predicta Pistorii et omnis eius comitatus atque districtus sit et esse intelligatur verum et originale territorium atque comitatus de territorio et comitatu civitatis Florentie et quarterio Sancte Marie Novelle et vexillo Unicorni dicte civitatis ${ }^{32}$.

Il 10 settembre 1401 entrarono in Pistoia truppe mercenarie al soldo della dominante, e i due ambasciatori fiorentini Matteo di Niccolò Strozzi e Lorenzo di Filippo Machiavelli invitarono il comune di Pistoia a cedere per sempre a Firenze la completa sovranità sulla città e sul territorio ${ }^{33}$. Sotto la minaccia delle armi sia l'esecutivo che il Consiglio del comune furono costretti ad approvare la proposta fiorentina, mentre il notaio rogante il verbale della riunione consolare, ser Agapito da Poppi, annotava a margine del testo un malinconico: «Heu, heu» ${ }^{34}$, e il cronista Luca Dominici, partecipante alla stessa riunione, ne chiudeva il racconto con un emblematico: «Christo ci aiuti» ${ }^{35}$.

La rivolta pistoiese del 1400-01 fu così, per Firenze, anche l'occasione diretta per imporre il proprio dominio e la propria sovranità su Pistoia, e forse la rottura stessa degli equilibri istituzionali costruiti nei precedenti decenni sarà stata salutata con favore dai fiorentini, come circostanza propizia per consolidare e dare sanzione definitiva al loro potere su Pistoia. La guerra civile raggiunse comunque il suo apice tra l'agosto e il settembre 1401 con incendi, saccheggi e violenze che agitarono l'intero territorio pistoiese, mentre in seguito all'intervento fiorentino i disordini e gli scontri proseguirono soprattutto nel contado e nella montagna pistoiese, ove Riccardo Cancellieri, asserragliato nella rocca della Sambuca, continuava a colpire con violente incursioni tutti i castelli limitrofi. A partire da metà dicembre Firenze assunse il controllo delle entrate pistoiesi e riformò completamente la costituzione del comune. Il 19 gennaio 1402 il contado di Pistoia fu suddiviso in quattro podesterie (Montale, Tizzana, Serravalle e Larciano), cui si aggiungeva il Capitanato della montagna ${ }^{36}$. L'intervento fiorentino non riuscì però a risolvere i conflitti

31 FIORAVANTI, 1968, Appendice: 57-60, per la trascrizione del diploma imperiale.

32 ASF, Capitoli, 54, c. 15v, 3 dicembre 1401. Copia della provvisione in ASF, Capitoli, 55 , c. $2 \mathrm{r}-\mathrm{v}$.

33 NERI, 1999: 21. Cronache di ser Luca Dominici, 1933-1939, vol. 2: 43-44.

34 ASPt, Comune, Provvisioni, 27, cc. 45r-47r.

35 Cronache di ser Luca Dominici, 1933-1939, vol. 2: 45.

36 Cronache di ser Luca Dominici, 1933-1939, vol. 2: 58-64, 69. 
e i disordini che si protrassero fino all'ottobre $1403^{37}$, quando fu possibile giungere ad una generale pacificazione delle parti.

Riguardo all'istituzionalizzazione del bipartitismo e all'equilibrio cercato da Firenze nella gestione della vita politica pistoiese afferma molto efficacemente Stephen Milner:

Solo due volte nel corso del secolo XV tale fragile equilibrio fu spezzato. Questi due episodi, che si collocano proprio all'inizio e alla fine del Quattrocento, sono di cruciale importanza per comprendere la natura mutevole dell'equilibrio raggiunto nel corso del secolo. La crisi del 1401 fu l'esito delle ansie fiorentine per la fedeltà della fazione dei Cancellieri di fronte all'aggressione viscontea, mentre la discordia civile che consumò Pistoia dopo il $1498 \mathrm{fu}$ in chiara relazione con l'esilio dei Medici quattro anni prima ${ }^{38}$.

Nonostante, infatti, già a partire dal primo Quattrocento fossero attive in Pistoia reti clientelari e di patronato per mezzo delle quali alcuni eminenti cittadini fiorentini si fecero arbitri delle parti pistoiesi e della vita politica della città (primi tra tutti Puccio Pucci, commissario fiorentino vicino ai Panciatichi e Neri di Gino Capponi, rettore, commissario, riformatore degli statuti pistoiesi e molto vicino alla famiglia Cancellieri, che alla sua morte ottenne il titolo di «protector et pater civitatis Pistorii») ${ }^{39}$, fu con l'emergere della figura di Cosimo de' Medici che si stabilì di fatto un nuovo equilibrio politico destinato a durare fino al 1494. La solidità della posizione di potere assunta in Firenze dopo il 1434 consentì a Cosimo de' Medici di acquisire sempre più influenza sulle parti pistoiesi, fino a che la riforma generale degli uffici del 1458, approvata soltanto quattro mesi dopo la morte di Neri di Gino Capponi con il consenso dello stesso Cosimo, sancì la soppressione del sistema bipartitico eliminando la suddivisione delle borse tra Panciatichi e Cancellieri ${ }^{40}$.

\section{IL 'MONOPOLIO’ MEDICEO DEL PATRONATO PISTOIESE}

L'abolizione del regime bipartitico, che segnò il passaggio ad un nuovo tipo di governo della vita politica pistoiese da parte di Firenze, maggiormente improntato alla dimensione clientelare, privatistica ed informale di gestione del potere, fu incoraggiata anche dagli scontri di fazione del $1455^{41}$, che co-

37 Cronache di ser Luca Dominici, 1933-1939, vol. 2: 175, 201.

38 MILNER, 2002: 408.

39 CONNELL, 53 (1991): 534-539. NERI, 1999: 40-45.

40 ASF, Statuti delle comunità autonome e soggette, 595, cc. 287r-303r.

41 SOZOMENO, 1908: 52. COLUCCI, 1752: 287-297. FIORAVANTI, 1968: 355-356. NERI, 1999: 47-49. 
strinsero l'autorità fiorentina ad un altro deciso intervento. Il 22 aprile 1455 furono, infatti, approvati i Capitoli dei 'Paciali', ordinamenti speciali voluti dalla dominante per pacificare le parti pistoiesi ${ }^{42}$, che prevedevano sia l'estensione dell'immunità a chi avesse sodato la pace entro sei mesi, sia la promozione di misure punitive per scongiurare il ripetersi di simili disordini ${ }^{43}$.

La volontà politica dell'autorità fiorentina poté a questo punto essere interamente ridisegnata sopra l'influenza che i Medici esercitavano su Pistoia. Cosimo, dagli anni cinquanta del Quattrocento unico arbitro delle dinamiche di potere delle parti pistoiesi, vide nell'abolizione delle istituzioni bipartitiche la possibilità di governare direttamente la città attraverso la fitte rete clientelare ordita nei due decenni precedenti, così come era stato capace di fare nella sua Firenze. Scrive Milner:

Mentre la supremazia medicea all'interno di Firenze si era consolidata attraverso la sistematica manipolazione delle procedure elettorali cittadine, l'influenza della famiglia negli affari pistoiesi si era affermata attraverso l'uso del patronato come strumento di governo ${ }^{44}$.

Il nuovo indirizzo politico inaugurato da Cosimo verrà portato a perfezione in epoca laurenziana, quando il Magnifico riuscirà a diventare tutore unico e patrono diretto dell'intera comunità pistoiese, elidendo sostanzialmente il ruolo e il potere delle parti.

Come ampiamente documentato dall'epistolario laurenziano ${ }^{45}$, il Magnifico intratteneva rapporti con numerose famiglie pistoiesi, indipendentemente dagli schieramenti di fazione (Rossi, Ippoliti, Taviani, Melocchi, Panciatichi, Bracciolini e Cancellieri). Egli favorì sempre i ceti eminenti cittadini, tutelandone gli interessi contro quelli degli abitanti delle comunità del contado ${ }^{46}$. Questa inversione di tendenza nelle relazioni tra Firenze e Pistoia si concretizzò nei fatti nella concessione di ampi privilegi (che per altro perdureranno ancora nei successivi due secoli sotto i granduchi medicei), come una particolare autonomia dei pistoiesi da alcune magistrature fiorentine quali i Cinque del contado, i Conservatori delle leggi, i Regolatori delle entrate e uscite e gli Ufficiali di torre ${ }^{47}$. Il Magnifico si dimostrò anche attivo come arbitro e pacificatore delle parti e dei contrasti tra le famiglie eminenti: nel 1471, ad esempio, grazie al suo intervento fu possibile sanare gli attriti tra Antonio Can-

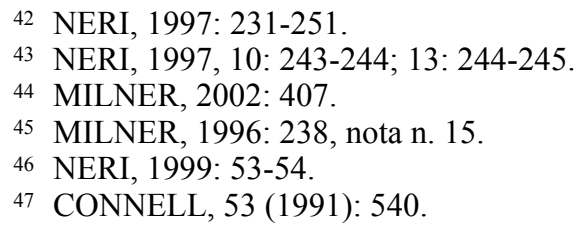


cellieri, Niccolò Bracciolini e Lorenzo ed Angelo della Stufa ${ }^{48}$. Certo le fazioni di Pistoia non scomparvero nel periodo laurenziano, ma, mentre i conflitti riguardavano più da vicino gli uomini appartenenti alle due parti, i capi delle grande famiglie si dimostravano particolarmente uniti nel tentativo di essere reintrodotti negli uffici e di ottenere riduzioni fiscali, grazie alle rispettive alleanze fiorentine. In occasione delle tre riforme delle modalità di accesso agli uffici pubblici (1474, 1477 e 1487), Panciatichi e Cancellieri cercarono in ogni modo, sempre senza successo, di essere riammessi alla cariche politiche sfruttando l'intercessione di Lorenzo de' Medici ${ }^{49}$.

Il fatto che la città di Pistoia fosse ora totalmente dipendente dalla volontà e dai maneggi del Magnifico, contribuì ad indebolire ulteriormente il regime politico pistoiese. La morte di Lorenzo nel 1492 e la cacciata di Piero de' Medici da Firenze nel 1494 lasciarono in Pistoia un tale vuoto di potere, che lo scontro di fazione riesplose qualche anno dopo ancora più feroce e cruento di prima. Scrive a questo proposito William Connell:

Dopo la fine di una struttura che istituzionalizzava la divisione fra le due parti, e in assenza di una gestione centralizzata del patronato, questa rivalità divenne sempre più aperta, coinvolgendo anche i politici fiorentini, fino alla catastrofica guerra civile degli anni 1499-1502, che sarebbe costata la vita a circa 2.500 pistoiesi fra cittadini e contadini ${ }^{50}$.

Lo stesso Connell ravvisa nel periodo che si apre all'alba della cacciata dei Medici da Firenze il terzo ed ultimo momento della storia delle relazioni politiche con la città di Pistoia: ad una prima fase corrispondente all'istituzionalizzazione del bipartitismo (1376-1450 circa), e ad una seconda in cui si stabilisce una sorta di patronato unico mediceo (dagli anni cinquanta del Quattrocento), si giunge infine, a causa del vuoto di potere lasciato dai Medici, al riaccendersi di quelle lotte di fazione che condurranno alla guerra civile del 1499-1502.

Dopo il 1494 a Pistoia cominciarono lentamente a risvegliarsi le componenti antimedicee. I Consigli deliberarono di far rimuovere dal palazzo del comune le insegne e le armi dei 'privati cittadini' (cioè dei Medici) ${ }^{51}$, mentre fu deciso di inserire un grande emblema della città di Pistoia. Furono anche allontanati alcuni ufficiali di provata fede medicea, come apprendiamo dai

48 MILNER, 1996: 244.

49 NERI, 1999: 60. ASF, Mediceo avanti il Principato, 391, 16 maggio 1474, lettere indirizzate al Magnifico dai figli di Antonio Cancellieri che si lamentano delle promesse fatte, e poi non mantenute, a loro e a Bastiano Panciatichi.

50 CONNELL, 53 (1991): 542.

51 ASPt, Comune, Provvisioni, 50, c. 60v, 28 novembre 1494. 
Ricordi di Francesco Ricciardi ${ }^{52}$. I nuovi Capitoli tra Firenze e Pistoia approvati nel $1496^{53}$ consentirono al patriziato pistoiese di ottenere un regime di particolare autonomia dai fiorentini ${ }^{54}$. I Panciatichi, molto favoriti negli ultimi anni dal regime mediceo, subirono ovviamente il ritorno e l'ascesa della parte cancelliera, cui il governo repubblicano di Firenze sembrò incline a concedere il favore nelle nuove contese ${ }^{55}$. Le tensioni che alimentavano la vita politica fiorentina all'indomani della restaurazione della repubblica venivano di fatto replicate all'interno di Pistoia, a causa dei forti legami clientelari instauratisi nel corso del Quattrocento. Afferma Stephen Milner:

In questo caso, la mancanza di una risposta unitaria da parte di Firenze significò che le divisioni pistoiesi furono esacerbate dalle tensioni all'interno del 'reggimento' fiorentino, come hanno sottolineato Guicciardini nelle Storie fiorentine, Niccolò Valori nelle sue Ricordanze e Pietro Vaglienti nella Storia dei suoi tempi ${ }^{56}$.

Il conflitto riesplose feroce dopo il 1498, ricalcando gli eventi della guerra civile di un secolo prima. Questa volta furono i Panciatichi ad essere espulsi ed esiliati da Pistoia dai Cancellieri, ma anche in questo caso gli scontri e le violenze divamparono in gran parte del contado e della montagna pistoiese. La repubblica fiorentina, minacciata all'inizio del nuovo secolo dalle mire espansionistiche del Valentino e impegnata nella nuova fase della guerra contro Pisa, procedette con ben poca solerzia nei confronti dei gravi disordini pistoiesi, almeno fino a quando, nell'estate del 1502, un'altra pericolosa rivolta, quella di Arezzo e della Valdichiana, fomentata proprio da Cesare Borgia e da uno dei suoi luogotenenti (Vitellozzo Vitelli), sembrò mettere in pericolo la sopravvivenza stessa del dominio territoriale fiorentino. Questa politica temporeggiatrice dovette, però, apparire in tutta la sua cinica crudeltà a quei pistoiesi che ne subirono le conseguenze sulla propria pelle, come ci testimonia l'acre risentimento della prosa storica di un erudito pistoiese del Settecento, che torna più di duecento anni dopo a condannare il vile opportunismo della dominante:

[...] e i Fiorentini, che come Vicarj Imperiali avevano avuto di Pistoja il governo da Roberto Imperatore, trascurarono ora con vituperio, e biasimo di loro stessi

52 Ricordi storici di Francesco Ricciardi detto «Ceccodea», 1934: 56.

53 ASF, Provvisioni, 187, cc. 74v-76v, 31 ottobre 1496; copia in ASF, Statuti delle comunità autonome e soggette, $598, \mathrm{cc} .2 \mathrm{r}-6 \mathrm{v}$. ASF, Statuti delle comunità autonome e soggette, 598 , cc. $7 \mathrm{r}-8 \mathrm{v}, 3$ novembre 1496.

54 CONNELL, 53 (1991): 527-528. NERI, 1999: 62-63.

55 CONNELL, XCV (1993): 23-39. BUONI, ms. in BFP, Fondo Forteguerriano, C 225.

56 MILNER, 2002: 425. MELOCCHI, ms. in BMV, Manoscritti, CL, It., VI 197 (5803). Manoscritto originale in ASF, Acquisti e doni, 8. 
di porre un salutevole rimedio a tanti mali, per giugnere a godere una volta le sostanze de' Pistojesi, e per rendere i medesimi così stanchi dalle discordie più facili a tollerare il loro governo ${ }^{57}$.

\section{Firenze e Pistoia: interessi Particolari, Clientele e CONDizio- NAMENTI RECIPROCI}

Gli studi dedicati da William Connell, tra la fine degli anni ottanta e l'inizio degli anni novanta del secolo scorso, alla realtà di Pistoia ${ }^{58}$ hanno avuto il merito di fare luce sulle complesse dinamiche, politiche e clientelari, che avevano contraddistinto le relazioni tra Firenze e Pistoia nei molteplici ambiti della negoziazione politica, della formazione di reti di patronato e dei reciproci condizionamenti delle élites cittadine, della polarizzazione delle fazioni in lotta e del 'monopolio' mediceo imposto dalla metà del Quattrocento alla gestione informale della vita politica cittadina. Tutto questo, seguendo il suggerimento formulato da Gene Brucker negli anni sessanta del novecento ${ }^{59}$, quando egli invitava a considerare come tipico della società fiorentina tra tardo Trecento e primo Quattrocento un nuovo tipo di patronato, che aveva di fatto soppiantato i modelli corporativi tipici del comune delle arti e proposto dinamiche simili, per molti rispetti, ai legami di tipo feudale.

Diversamente da quanto accaduto per altre comunità dello stato territoriale fiorentino la città di Pistoia, ancora alla metà del Quattrocento, poteva vantare una condizione di particolare autonomia, sancita di fatto dalle riforme del dicembre $1454^{60}$ in base alle quali nessun cittadino o comitatino di Pistoia avrebbe potuto essere chiamato in giudizio a Firenze da alcune magistrature fiorentine operanti sul territorio (Cinque del contado, Conservatori delle leggi, Regolatori delle entrate e uscite, Ufficiali di torre e Ufficiali di notte). La vita politica pistoiese si affrancava in questo modo dall'ingerenza delle istituzioni fiorentine deputate al controllo del territorio ${ }^{61}$. Anche con le riforme del $1496^{62}$ il patriziato pistoiese poté ottenere altre importanti concessioni: tutte le leggi che in futuro Firenze avrebbe promulgato per la gestione del dominio territoriale non avrebbero dovuto applicarsi alla città di Pistoia e al suo contado, qualora ciò non fosse espressamente menzionato.

57 FIORAVANTI, 1968: 390.

58 CONNELL, 1989; 53 (1991): 523-543.

59 BRUCKER, 1 (1964): 2-11.

60 ASF, Statuti delle comunità autonome e soggette, 598, c. 28r-v, 19 dicembre 1454.

61 CONNELL, 53 (1991): 526.

62 Vedi supra, nota n. 53. 
I ceti eminenti pistoiesi ebbero perciò modo di ampliare molto la loro sfera d'influenza ${ }^{63}$, mentre la struttura portante delle istituzioni fiorentine allentava la presa sul territorio pistoiese. Quello che, però, Firenze riuscì a mantenere in modo energico fu il potere esecutivo: i rettori fiorentini videro infatti accresciuto il peso determinante delle loro funzioni a partire dal $1455^{64}$. Si stabilì così, dal secondo Quattrocento, «quell'equilibrio fra le larghe autonomie concesse localmente sul piano della gestione ordinaria e le forti prerogative esercitate da Firenze a livello del potere esecutivo che caratterizzerà la situazione di Pistoia nello stato toscano nei secoli successivi» ${ }^{65}$. I rapporti clientelari e di patronato che legavano il ceto eminente pistoiese alle principali famiglie fiorentine costituivano, di fatto, il terzo livello su cui venivano ad innestarsi le complesse dinamiche politiche in atto tra dominante e città soggetta. In realtà i cosiddetti legami di 'amicizia' erano ben più antichi dei processi di formazione dello stato territoriale fiorentino e affondavano le proprie radici nell'epoca delle lotte di fazione tra Bianchi e Neri66. Come abbondantemente testimoniato dalle cronache coeve ${ }^{67}$, fu però a partire dal 1375 che cominciarono a stabilirsi solide alleanze parentali, per mezzo di importanti matrimoni come quelli che legarono i Panciatichi agli Albizzi.

Il più influente patrono fiorentino di Pistoia nel primo Quattrocento fu Neri di Gino Capponi68. Il fatto che il Capponi si trovasse a confermare l'istituzionalizzazione delle parti pistoiesi riuscì a garantirgli il riconoscimento e l'appoggio di entrambe le famiglie di Panciatichi e Cancellieri, nonostante egli rimanesse nei suoi affari maggiormente legato a questi ultimi69. Con l'emergere della preminenza medicea l'atteggiamento nei confronti delle fazioni pistoiesi si trovò di fatto a ricalcare gli equilibri politici e le dinamiche di potere che accompagnavano la vita pubblica fiorentina. Inizialmente, Cosimo, per bilanciare le alleanze degli Albizzi con i Panciatichi, si avvicinò alla parte cancelliera; in seguito, però, quando nel 1458 la costituzione bipartitica di Pistoia venne abolita, egli cominciò ad intrattenere rapporti anche con la parte panciatica. Appare manifesto, perciò, che i Medici volessero assurgere al ruolo di patroni unici di entrambe le parti pistoiesi ${ }^{70}$.

63 CONNELL, 53 (1991): 530-531.

64 PAOLI, I (1899): 11-24. NERI, 1997: 231-251.

65 CONNELL, 53 (1991): 532.

66 DAVIDSOHN, 1972-1973², vol. 4: 181-182.

67 Cronache di ser Luca Dominici, 1933-1939, vol. 2: 9-11.

68 CONNELL, 53 (1991): 535-537.

69 CONNELL, 53 (1991): 537.

70 «Sembrerebbe evidente che i Medici pensassero di poter gestire le cose pistoiesi senza rispettare le tradizionali divisioni della tarda età comunale. Il coronamento di questa strategia clientelare rivolta verso entrambe le parti di Pistoia fu raggiunto nel 1478, quando quasi subito dopo la morte in esilio a Lucca di Gualtieri Panciatichi, Lorenzo de' Medici e la sua famiglia 
Per mettere in rilievo il cambiamento radicale imposto dai Medici alle modalità di governo della vita politica pistoiese, anche Stephen Milner sottolinea come gli ufficiali e i commissari fiorentini svolgessero ancora le loro specifiche mansioni,

\begin{abstract}
ma la responsabilità di mantenere l'equilibrio politico divenne sempre più un incarico dei Medici stessi, piuttosto che un obiettivo perseguito attraverso gli organi tradizionali di governo del territorio ${ }^{71}$.
\end{abstract}

In realtà il coinvolgimento dei Medici nella vita politica di Pistoia divenne significativo già a partire dal secondo quarto del secolo $\mathrm{XV}$, quando la supremazia medicea sulla città cominciava ad essere documentata nelle provvisioni e nei registri del comune ${ }^{72}$, a nome del quale si inviavano alla famiglia fiorentina regali, ambasciatori a fare gli onori cittadini quando i membri di essa giungevano in visita a Pistoia o nel suo territorio, o si stanziavano denari per la commemorazione dei defunti. Questo ossequio lo possiamo ritrovare anche nel registro linguistico utilizzato per descrivere i Medici quali patroni e protettori della città di Pistoia ed essa quale loro cliente ${ }^{73}$.

In riferimento all'Archivio Mediceo avanti il Principato, importante strumento per cogliere, attraverso l'esame della corrispondenza medicea, l'effettivo peso delle relazioni clientelari intessute dai Medici con le autorità pistoiesi e con i cittadini eminenti, Milner ha potuto identificare, su di un totale di 1.231 missive (tra il 1405 e il 1538), ben 600 lettere per il decennio 1470-80, 92 per quello 1490-1500, e 140 per il decennio 1510-2074. Durante la prima metà del periodo laurenziano, negli anni immediatamente precenti l'esilio del 1494 e in quelli successivi al ritorno del 1512, Milner nota un «marcato sviluppo» del volume della corrispondenza inviata da Pistoia. Lorenzo è, ovviamente, il più cercato, con un totatle di 669 lettere (vale a dire più della metà dell'intera corrispondenza). È importante notare che scrivono ai Medici i membri eminenti di entrambe le fazioni pistoiesi, per assicurarsi il loro appoggio nei tentativi di riforma delle procedure elettorali, per denunciare eventuali offese o maltrattamenti ricevute dagli aderenti all'opposta fazione; lo stesso comune poteva rivolgersi ai Medici per promuovere gli interessi della città nei confronti del contado o per cercare un solido alleato che pero-

accettarono l'ospitalità dei figli di Gualtieri nel palazzo Panciatichi a Pistoia»: CONNELL, 53 (1991): 539.

71 MILNER, 2002: 419.

72 MILNER, 2002: 420. ASPt, Consigli, provvisioni e riforme, 42, c. 46r; 44, c.74r; 48, c. 344 r.

73 MILNER, 2002: 420-421.

74 MILNER, 2002: 422-423. 
rasse la causa cittadina dinanzi alle istituzioni fiorentine maggiormente esigenti e pressanti ${ }^{75}$.

La questione del 'monopolio' mediceo sul patronato pistoiese viene sollevata anche da Milner, il quale torna ad affermare come l'esilio del 1494 creasse, in Pistoia come in Firenze, un vero e proprio 'stato di sospensione':

Le lotte per la supremazia all'interno del 'reggimento' fiorentino erano replicate all'interno di Pistoia, come conseguenza dei legami patronali e di parentela tra le due città. La disunità in una di esse alimentava la disunità nella sua vicina ${ }^{76}$.

Il fatto che il Magnifico, poi, avesse favorito la parte panciatica, aveva contribuito a marginalizzare il ruolo dei Cancellieri all'interno della vita politica pistoiese già a partire dai primi anni settanta del Quattrocento, portandosi dietro rancori personali, esclusioni dagli uffici, privazioni di patrimoni ecc. Per riequilibrare tale situazione sarebbero serviti o l'intervento di un potere centrale forte o la presenza di un referente unico del patronato territoriale, ma entrambi questi fattori erano venuti a mancare dopo il 149477 .

Da questo punto di vista, molto interessante appare un altro lavoro di Connell ${ }^{78}$ teso ad indagare il modo in cui i nuovi equilibri politici della Firenze post 1494 si trovarono a condizionare la vita delle fazioni pistoiesi, con la creazione di nuove alleanze politiche o con il rafforzamento dei legami già esistenti tra le parti di Pistoia e le famiglie dei patrizi fiorentini, tornati al centro della scena pubblica dopo l'esilio mediceo. In un passo delle Storie fiorentine il Guicciardini, discutendo il rapporto di Pistoia con Firenze durante la guerra civile del 1499-1502, menziona le famiglie fiorentine che si trovarono

75 MILNER, 2002: 424.

76 MILNER, 2002: 425.

77 «In questo caso, però, il processo di reintegrazione si dimostrò più difficile, per la mancanza di un potere coercitivo in grado di sanzionare i capitoli. [...] L'esilio dei Medici nel 1494 provocò un vuoto 'patronale' a Pistoia, lasciando la città alla ricerca di un nuovo centro carismatico. L'effetto di assegnare privilegi al comune pistoiese fu di invertire il processo di burocratizzazione fiorentino nei confronti di Pistoia, finendo così con l'indebolire il meccanismo delle regole e del rispetto per le istituzioni formali di governo. La mancanza di rispetto nei confronti di una struttura comunale chiaramente articolata e costituita, unita al peso delle parti rispettive e alla natura della divisione storica, precluse la possibilità di affermazione egemonica di un gruppo sull'altro e, allo stesso tempo, la realizzabilità anche della finzione del consenso. Di conseguenza, ciascuno puntò a legittimarsi attraverso l'occupazione fisica del comune e l'esclusione degli avversari da ogni condivisione nel governo e nella gestione del patrimonio civico. Nel caso di Pistoia, quindi, la fazione che rimase all'interno della città rappresentò il comune da sola, in termini di mera occupazione del territorio»: MILNER, 2002: 426-427.

78 CONNELL, 1994a, vol. 1: 118-147. 
ad appoggiare la parte cancelliera ${ }^{79}$. Lauro Martines aveva notato per primo questo importante passaggio guicciardiniano ${ }^{80}$, e Connell torna significativamente a porvi attenzione per mettere in luce come i maggiori cittadini fiorentini fossero sempre stati «fautori» delle parti pistoiesi, e come il vuoto di potere causato dall'esilio mediceo avesse spinto i patrizi fiorentini a cimentarsi ulteriormente nel perseguimento di tali legami clientelari, per ristabilire una sorta di controllo politico sopra Pistoia ${ }^{81}$.

Che molte delle nuove alleanze si polarizzassero rispetto al fronte antimediceo e antivitellesco è piuttosto chiaro: diventare 'fautore' dei Cancellieri significava in questo senso ribadire la propria ostilità ai Medici e ai Vitelli, legati anche tramite vincoli parentali alla parte panciatica. Tale comportamento, però, non fece altro che esarcerbare gli animi dei membri delle fazioni pistoiesi, ed è proprio lo stesso Guicciardini a ritenere che buona parte della colpa per il nuovo divampare dei disordini civili spettasse proprio a eminenti fiorentini quali Bernardo Rucellai e Guidantonio Vespucci, a causa del loro fin troppo esplicito sostegno alla parte cancelliera nella vicenda che, nell' autunno-inverno 1498-99, cosituì il casus belli della futura guerra civile: la nomina alla carica vacante di spedalingo di San Gregorio ${ }^{82}$. Come ricostruito da Connell con dovizia di particolari, fu proprio l'intervento del Rucellai in favore del suo cliente di parte cancelliera, Bernardo Nutini, a consentire al vescovo Pandolfini (anch'egli di parte cancelliera) di farlo eleggere formalmente come spedalingo ${ }^{83}$.

E nel momento in cui la Signoria di Firenze, che era stata interpellata per risolvere la questione dopo che i Panciatichi avevano occupato l'ospedale di San Gregorio non riconoscendo la nomina del vescovo, dovette pronunciarsi riguardo all'elezione, un peso determinante in tale decisione dovette senz'altro spettare all'allora Gonfaloniere di giustizia fiorentino, che altri non era che il più fidato collaboratore del Rucellai, Guidantonio Vespucci. Il 20 gennaio

79 «Avevano e' Cancellieri moltissimi fautori: una parte naturalmente; una parte di quegli erano stati inimici de' Medici, e' quali odiavano e' Panciatichi perché Lorenzo e la casa de' Medici gli aveva sempre favoriti; una parte di quegli erano stati inimici de' Vitelli, perché una sorella di Paolo e di Vitellozzo era maritata a uno figliuolo di Niccolaio Bracciolini, uno de' capi panciatichi, e per questo rispetto e' Vitelli avevano sempre dato favore a quella parte. Eranne capi messer Guidantonio Vespucci, Bernardo Rucellai, messer Francesco Gualterotti, Giovan Batista Ridolfi, Guglielmo de' Pazzi, e' Nerli, Lorenzo di Pierfrancesco, Luca d'Antonio degli Albizzi, Iacopo Pandolfini; de' quali, Giovan Batista Ridolfi se ne portò sempre costumatissimamente, messer Guido e Bernardo Rucellai se ne scopersono in modo che n'ebbono grandissimo carico, e fu dal popolo imputato a loro in gran parte questo disordine»: GUICCIARDINI, $2006^{2}$, cap. XX: 333.

80 MARTINES, 1968: 234-237.

${ }^{81}$ CONNELL, 1994a, vol. 1: 118-119.

82 CONNELL, 1994a, vol. 1: 130-131.

$83 \ll[\ldots]$ an attempt to legitimize an electoral abuse»: CONNELL, 1994a, vol. 1: 131. 
1499, così, la Signoria rendeva noto di approvare l'elezione di Bernardo Nutini come spedalingo di San Gregorio ${ }^{84}$. Non si può, allora, non tenere in debita considerazione quanta parte ebbero nel riaccendersi delle lotte di fazione pistoiesi le nuove strategie politico-clientelari delle eminenti famiglie fiorentine, nel momento in cui il vuoto di potere mediceo doveva essere colmato attraverso la definizione di nuovi assetti ed equilibri politici, tanto nelle questioni interne al reggimento fiorentino quanto in quelle che riguardavano una comunità soggetta ormai inscindibilmente legata agli umori e ai destini della dominante.

Com'è ovvio supporre, anche i Panciatichi disponevano di importanti 'fautori' in Firenze ${ }^{85}$, persino dopo il crollo del regime mediceo. Connell ricostruisce, anche in questo caso, i legami stretti durante il XV secolo dai Soderini, dai Guicciardini e dai Salviati con la parte panciatica ${ }^{86}$, per poi passare ad analizzare una fonte molto utile per la determinazione delle strategie clientelari delle élites fiorentine durante la guerra civile pistoiese del 1499-1502: le Consulte e pratiche della repubblica fiorentina ${ }^{87}$. Anche in questo caso era stato Lauro Martines, cogliendo il suggerimento espresso dal Guicciardini, ad affermare come i registri delle pratiche della repubblica fiorentina avrebbero potuto dare indicazioni notevoli relativamente agli schieramenti dei notabili fiorentini rispetto alle fazioni in guerra a Pistoia ${ }^{88}$. Non disponendo però di un resoconto preciso degli eventi in questione, Martines non era riuscito a collegare i punti di vista espressi nelle Consulte con la presunta appartenenza politica dei partecipanti, cosa che Connell è invece riuscito a fare prendendo in esame le pratiche che ebbero luogo nell'agosto del 1500, dal momento che, in precedenza, gli affari pistoiesi non vi avevano trovato posto e, successivamente alla primavera del 1501, la maggior parte degli oligarchi fiorentini più vicini ai Cancellieri cessarono quasi interamente di partecipare alle attività ufficiali delle istituzioni fiorentine.

Nelle riunioni di queste Consulte emerge piuttosto chiaramente l'interesse dei 'fautori' dei Cancellieri affinché la Signoria intervenga con decisione per

$84 \ll[\ldots]$ precipitating the factional violence that would engulf Pistoia for the next four years. Small wonder that the Florentine popolo blamed Rucellai and Vespucci for the disorder»: CONNELL, 1994a, vol. 1: 131. Ricordi storici di Francesco Ricciardi detto "Ceccodea», 1934: 70.

85 «Gli amici de' Panciatichi erono in minore numero ed anche andavano lentamente, e ne erano quasi capi Piero Soderini, Piero Guicciardini, Alamanno ed Iacopo Salviati, e' quali non si scoprivano molto e procedevano con rispetto»: GUICCIARDINI, 2006 ${ }^{2}$, cap. XX: 332. MARTINES, 1963: 63-65.

86 CONNELL, 1994a, vol. 1: 132-136.

87 ASF, Consulte e pratiche, 66 (13 maggio 1500 - 31 maggio 1502) e 67 (3 giugno 1502 15 maggio 1505), passim. Si vedano anche i documenti editi in: Consulte e pratiche della Repubblica fiorentina, 1498-1505, 1993, voll. 1-2: 357-784, passim; vol. 2: 785-1032, passim. Vedi CONNELL, 1989: 200-356.

${ }^{88}$ CONNELL, 1994a, vol.1: 136-137. 
porre fine ai disordini pistoiesi. Durante la Signoria del bimestre luglio-agosto 1500, Piero Panciatichi (appartenente al ramo fiorentino della famiglia e legato anche da vincoli di parentela allo stesso Gonfaloniere di giustizia), era infatti riuscito ad aiutare la propria parte in Pistoia, facendo in modo che le autorità fiorentine non intervenissero contro i Panciatichi i quali, disponendo di un numero maggiore di uomini, se non ostacolati avrebbero potuto risolvere le contese a loro favore ed imporsi sui Cancellieri. Per questo motivo, notabili come Guidantonio Vespucci consigliavano l'uso della forza per riportare l'ordine in Pistoia (a favore ovviamente della parte cancelliera) ${ }^{89}$, mentre i 'fautori' dei Panciatichi, come Piero Soderini, spingevano per un intervento più 'istituzionale', pur condividendo la necessità di inviare truppe militari ${ }^{90}$.

Quando, però, dopo l'invio di 200 soldati da parte di Firenze nel pomeriggio del 14 agosto 1500, nella notte seguente i Cancellieri riuscirono a riprendere terreno in città grazie all'aiuto di centinaia di armati provenienti dal bolognese, le posizioni dei patrizi fiorentini si invertirono: il Vespucci, prima deciso promotore di un intervento armato, divenne più cauto, mentre il Soderini chiedeva celerità nelle risoluzioni della Signoria ${ }^{91}$. Così, come ha bene illustrato Connell, gli interessi particolari che legavano gli oligarchi fiorentini a ciascuna delle fazioni pistoiesi e il disaccordo sulle possibili soluzioni da adottare ebbero l'effetto di ritardare ulteriormente l'azione univoca della Signoria, di modo che la crisi dell'agosto 1500 degenerò in una vera e propria guerra civile che si protrasse per altri due anni mietendo centinaia di vittime ${ }^{92}$.

\section{BibliOgRAFÍA}

Brucker, Gene A., «The Structure of Patrician Society in Renaissance Florence», Colloquium, 1 (1964): 2-11.

Capitoli del Comune di Firenze, Inventario e regesto, 2 voll., I, a cura di C. Guasti, Firenze, Cellini, 1866; II, a cura di A. Gherardi, Firenze, Cellini, 1893.

Cherubini, Giovanni, «Apogeo e declino del comune libero», in G. Cherubini (a cura di), Storia di Pistoia, II. L'età del libero Comune. Dall'inizio del XII alla metà del XIV secolo, Firenze, Le Monnier, 1998: 41-88.

89 ASF, Consulte e pratiche, 66 , c. $87 \mathrm{r}$ (3 agosto 1500$)$ e c. $92 \mathrm{v}$ (14 agosto 1500$)$. Vedi Consulte e pratiche della Repubblica fiorentina, 1498-1505, 1993, vol. 1: 426-427, 431-432.

90 ASF, Consulte e pratiche, 66, c. 93r-v (14 agosto 1500). Vedi Consulte e pratiche della Repubblica fiorentina, 1498-1505, 1993, vol. 1: 432.

91 ASF, Consulte e pratiche, 66, cc. 97v-100r (18 agosto 1500). Vedi Consulte e pratiche della Repubblica fiorentina, 1498-1505, 1993, vol. 1: 436-441.

92 CONNELL, 1994a, vol. 1: 141. 
Colucci, Benedetto, Lazareus, in F.A. Zacharia, Bibliotecha pistoriensis, Torino, Augustae Taurinorum, 1752: 287-297.

Connell, William J., "“I fautori delle parti”. Citizen Interest and the Treatment of a Subject Town, c. 1500», in C. Lamioni (a cura di), Istituzioni e società in Toscana nell'età moderna, Atti delle giornate di studio dedicate a Giuseppe Pansini (Firenze, 4-5 dicembre 1992), 2 voll., Roma, Ministero per i beni culturali e ambientali, Ufficio centrale per i beni archivistici, 1994a, vol. 1: 118-147.

Connell, William J., «Changing Patterns of Medicean Patronage. The Florentine Dominion During the Fifteenth Century, in G.C. Garfagnini (a cura di), Lorenzo il Magnifico e il suo mondo, Atti del convegno internazionale di studi (Firenze, 913 giugno 1992), Firenze, Olschki, 1994b: 87-107.

Connell, William J., «Clientelismo e Stato territoriale. Il potere fiorentino a Pistoia nel XV secolo», Società e Storia, 53 (1991): 523-543.

Connell, William J., «Il commissario e lo Stato territoriale fiorentino», Ricerche storiche, XVIII (1988): 591-617.

Connell, William J., «Un cronista sconosciuto del primo '500: Bastiano Buoni e la sua cronaca De’ casi di Pistoia», Bullettino Storico Pistoiese, XCV (1993): 23-39.

Connell, William J., Republican Territorial Government: Florence and Pistoia in the Fifteenth and Early Sixteenth Century, Ph.D. diss., Berkeley, University of California, 1989.

Consulte e pratiche della Repubblica fiorentina, 1498-1505, a cura di D. Fachard, 2 voll., Genève, Droz, 1993.

Cronache di ser Luca Dominici, a cura di G.C. Gigliotti, 2 voll., Pistoia, Pacinotti, 1933-1939.

Davidsohn, Robert, Storia di Firenze, trad. it., 8 voll., Firenze, Sansoni, 1972-1973².

De Angelis, Laura, «La fine della libertà pistoiese», in L. Borgia et alii (a cura di), Studi in onore di Arnaldo D'Addario, 4 voll., Lecce, Conte Editore, 1995, vol. 4: 1157-1165.

Dedola, Marco, "“Tener Pistoia con le parti”. Governo fiorentino e fazioni pistoiesi all'inizio del '500», Ricerche Storiche, XXII (1992): 239-259.

Dedola, Marco, «Governare sul territorio. Podestà, capitani e commissari a Pistoia prima e dopo l'assoggettamento a Firenze (XIV-XVI secolo), in C. Lamioni (a cura di), Istituzioni e società in Toscana nell 'età moderna, Atti delle giornate di studio dedicate a Giuseppe Pansini (Firenze, 4-5 dicembre 1992), 2 voll., Roma, Ministero per i beni culturali e ambientali, Ufficio centrale per i beni archivistici, 1994, vol. 1: 215230 .

Fioravanti, Jacopo Maria, Memorie storiche della città di Pistoja, Lucca, Benedini, 1758 (ristampa anastatica: Bologna, 1968).

Fubini, Riccardo, Antonio Ivani da Sarzana: un teorizzatore del declino delle autonomie comunali, in Egemonia fiorentina ed autonomie locali nella Toscana nordoccidentale del primo Rinascimento: vita, arte, cultura, Pistoia, Centro italiano di studi di storia e d'arte, 1978: 113-164. 
Gai, Lucia, «Centro e periferia: Pistoia nell'orbita fiorentina durante il '500», in Pistoia: una città nello stato mediceo, Pistoia, Edizioni del Comune di Pistoia, 1980: 9-147.

Guicciardini, Francesco, Storie fiorentine, a cura di A. Montevecchi, Milano, RCS Libri, $2006^{2}$.

Herlihy, David, Pistoia nel Medioevo e nel Rinascimento 1200-1430, Firenze, Olschki, 1972.

Kent, Dale V., «Dinamica del potere e patronato nella Firenze di Cosimo de' Medici», in I ceti dirigenti nella Toscana del Quattrocento, Firenze, Papafava, 1987: 49-62.

Kent, Dale V., The Rise of the Medici. Faction in Florence, 1426-1434, Oxford, Oxford University Press, 1978.

Le Consulte e Pratiche della Repubblica fiorentina nel Quattrocento (1401), I, a cura di E. Conti, Pisa, Giardini Editori, 1981.

Machiavelli, Niccolò, Il Principe, a cura di G. Inglese, Torino, Einaudi, 1995.

Martines, Lauro, Lawyers and Statecraft in Renaissance Florence, Princeton, Princeton University Press, 1968.

Martines, Lauro, The Social World of the Florentine Humanists, 1390-1460, Princeton, Princeton University Press, 1963.

Milner, Stephen J., «Capitoli e clienti a Pistoia nel secolo XV: dalle strutture repubblicane all'egemonia medicea», trad. it. di Patrizia Salvadori, in A. Zorzi e W.J. Connell (a cura di), Lo stato territoriale fiorentino (secoli XIV-XV). Ricerche, linguaggi, confronti, Atti del seminario internazionale di studi (San Miniato, 7-8 giugno 1996), Pisa, Pacini, 2002: 405-429.

Milner, Stephen J., «Lorenzo and Pistoia: Peacemaker or Partisan?», in M. Mallett and N. Mann (ed.), Lorenzo the Magnificent. Culture and Politics, London, The Warburg Institute, University of London, 1996: 235-252.

Neri, Francesco, «I Capitoli dei "Paciali" del 1455», in E. Vannucchi (a cura di), Pistoia e la Toscana nel Medioevo. Studi per Natale Rauty, Pistoia, Società pistoiese di storia patria, 1997: 231-251.

Neri, Francesco, «Società ed istituzioni: dalla perdita dell'autonomia comunale a Cosimo I», in G. Pinto (a cura di), Storia di Pistoia, III. Dentro lo Stato Fiorentino. Dalla metà del XIV alla fine del XVIII secolo, Firenze, Le Monnier, 1999: 1-80.

Paoli, Cesare, «I Capitoli dei "Paciali" di Pistoia del 1455 confermati dalla Signoria di Firenze nel 1473», Bullettino Storico Pistoiese, I (1899): 11-24.

Ricordi storici di Francesco Ricciardi detto «Ceccodea», a cura di A. Chiti, Pistoia, Pacinotti, 1934 («Rerum Pistoriensium Scriptores», 2).

Rubinstein, Nicolai, «Lorenzo de' Medici. The Formation of His Statecraft», in G.C. Garfagnini (a cura di), Lorenzo de' Medici. Studi, Firenze, Olschki, 1992, pp. 41-66.

Rubinstein, Nicolai, The Government of Florence Under the Medici, 1434-1494, Oxford, Clarendon Press, 1966; trad. it, Il governo di Firenze sotto i Medici (1434-1494), Firenze, La Nuova Italia, 1971. 
Salvadori, Patrizia, «I fiorentini e i centri del dominio», in A. Zorzi e W.J. Connell (a cura di), Lo stato territoriale fiorentino (secoli XIV-XV). Ricerche, linguaggi, confronti, Atti del seminario internazionale di studi (San Miniato, 7-8 giugno 1996), Pisa, Pacini, 2002: 477-497.

Salvadori, Patrizia, «Rapporti personali, rapporti di potere nella corrispondenza di Lorenzo dei Medici», in G.C. Garfagnini, Lorenzo il Magnifico e il suo tempo, Firenze, Olschki, 1992: 125-146.

Salvadori, Patrizia, Dominio e patronato. Lorenzo dei Medici e la Toscana nel Quattrocento, Roma, Edizioni di Storia e Letteratura, 2000.

Sozomeno, Chronicon Universale (1411-1455), a cura di G. Zaccagnini, in Rerum Italicarum Scriptores, XVI, I, Città di Castello, 1908: 1-54.

Zorzi, Andrea, «Giusdicenti e operatori di giustizia nello Stato territoriale fiorentino del XV secolo», Ricerche Storiche, XIX (1989): 517-552.

Zorzi, Andrea, «I Fiorentini e gli uffici pubblici nel primo Quattrocento: concorrenze, abusi, illegalità», Quaderni Storici, LXVI (1987): 725-751.

Recibido: $21 / 10 / 2013$

Aceptado: 08/09/2014 